\title{
Dietary fibre intake in relation to the risk of incident chronic kidney disease
}

\author{
Parvin Mirmiran ${ }^{1}$, Emad Yuzbashian $^{2}$, Golaleh Asghari ${ }^{2}$, Shaghayegh Sarverzadeh ${ }^{2}$ and Fereidoun Azizi $^{3}$ \\ ${ }^{1}$ Department of Clinical Nutrition and Dietetics, Faculty of Nutrition Sciences and Food Technology, National Nutrition and \\ Food Technology Research Institute, Shabid Beheshti University of Medical Sciences, 1416643931, Tehran, Iran \\ ${ }^{2}$ Nutrition and Endocrine Research Center, Research Institute for Endocrine Sciences, Shahid Beheshti University of Medical \\ Sciences, 1985717413, Tehran, Iran \\ ${ }^{3}$ Endocrine Research Center, Research Institute for Endocrine Sciences, Shabid Beheshti University of Medical Sciences, \\ 1985717413, Tehran, Iran
}

(Submitted 18 July 2017 - Final revision received 8 November 2017 - Accepted 28 November 2017 - First published online 21 January 2018)

\section{Abstract}

The purpose of this study was primarily to evaluate the association of total fibre intake with the risk of incident chronic kidney disease (CKD). We also evaluated the association of dietary fibre from fruits, vegetables, cereals and legumes with the incidence of CKD in a populationbased prospective study. We followed up 1630 participants of the Tehran Lipid and Glucose Study for $6 \cdot 1$ years, who were initially free of CKD. Baseline diet was assessed by a valid and reliable FFQ. Estimated glomerular filtration rate (eGFR) was calculated, using the Modification of Diet in Renal Disease Study equation, and CKD was defined as eGFR $<60 \mathrm{ml} / \mathrm{min}$ per $1.73 \mathrm{~m}^{2}$. OR using multivariable logistic regression was reported for the association of incident CKD with tertiles of dietary fibre intake. After adjustment for age, sex, smoking, total energy intake, physical activity, diabetes and using angiotensin-converting-enzyme inhibitor, the OR for subjects in the highest compared with the lowest tertile of total fibre intake was 0.47 (95\% CI $0.27,0.86)$. In addition, for every $5 \mathrm{~g} / \mathrm{d}$ increase in total fibre intake, the risk of incident CKD decreased by $11 \%$. After adjusting for potential confounders, OR for participants in the highest compared with the lowest tertile of fibre from vegetables was 0.63 (95\% CI $0.43,0.93)$ and from legumes it was 0.68 (95\% CI 0.47, 0.98). We observed inverse associations between total fibre intake and risk of incident $\mathrm{CKD}$, which demonstrate that high fibre intake, mainly from legumes and vegetables, may reduce the occurrence of CKD.

\section{Key words: Fruits: Vegetables: Legumes: Kidney function}

Chronic kidney disease (CKD) is a prevalent complex disease and is fast becoming a growing public health problem, leading to premature mortality or poor quality of life, exposing a heavy burden on healthcare systems ${ }^{(1,2)}$. Evidence available reveals that the global prevalence of CKD has a sharply increasing trend, affecting $10-15 \%$ of general populations ${ }^{(3,4)}$. Ageing, hypertension, diabetes, hyperlipidaemia, smoking and poor dietary intake have been identified as risk factors for CKD occurrence $^{(5,6)}$. Higher dietary intake of plant protein, vitamin C, $\mathrm{Mg}, \mathrm{K}, n-6$ fatty acids and lower intake of animal protein and $\mathrm{Na}$ are documented as the potential factors for prevention of CKD occurrence $^{(7,8)}$. Yet, dietary guidelines have not been developed for the prevention of kidney dysfunction.

Although a great deal of research has been conducted on the importance of dietary fibre intake in the prevention of CVD, diabetes and cancer, encouraging healthcare organisations to recommend dietary fibre intake ${ }^{(9,10)}$, findings on the dietary fibre and kidney function are limited and inconsistent ${ }^{(11-13)}$.
One cohort study indicates that subjects in the highest quartile of dietary cereal fibre, compared with those in the lowest, had a $50 \%$ decreased risk for incidence of CKD; however, total fibre intake was not associated with the occurrence of $\mathrm{CKD}^{(11)}$. Xu et $a l .{ }^{(12)}$ showed that higher total fibre intake was positively associated with estimated glomerular filtration rate (eGFR) among elderly men. In addition, greater dietary total fibre intake was associated with reduced risk of incident kidney stones in postmenopausal women ${ }^{(13)}$.

Previous studies on dietary fibre intake and kidney function have been conducted in developed countries. The Tehran Lipid and Glucose Study (TLGS) is a large prospective cohort study with different dietary habits required for substantiating and persuasion of preventive dietary recommendations. Current TLGS analyses showed that plant-based diets rich in fibre were related to a decrease in incident $\mathrm{CKD}^{(14,15)}$; total dietary fibre intake was also associated with reduced CVD risk ${ }^{(16)}$, and fruit fibre was associated with decreased the metabolic syndrome ${ }^{(17)}$.

Abbreviations: CKD, chronic kidney disease; eGFR, estimated glomerular filtration rate; TLGS, Tehran Lipid and Glucose Study.

* Corresponding author: G. Asghari, fax +98 21 22402463, email g_asghari@hotmail.com 
The purpose of this study was primarily to evaluate the association of total fibre intake with the risk of incident CKD. We also evaluated the association of dietary fibre from fruits, vegetables, cereals and legumes with the incidence of CKD in a population-based prospective study.

\section{Methods}

\section{Study population}

This study was conducted within the framework of the TLGS, an ongoing community-based prospective investigation aimed at preventing non-communicable diseases (NCD) by developing programmes promoting healthy lifestyles and reducing NCD risk factors in a sample of residents under coverage of three medical health centres in District No. 13 of Tehran, the capital city of Iran ${ }^{(18)}$. The baseline survey was a cross-sectional study conducted from 1999 to 2001, and surveys II (2002-2005), III (2006-2008), IV (2009-2011) and V (2012-2015) are prospective follow-up surveys.

From among the 12523 participants examined in the third survey of the TLGS, 3462 were randomly selected for dietary assessment, of whom 2417 participants were aged $\geq 27$ years. Subjects with a history of myocardial infarction or stroke due to the possibility of major changes in diet were excluded $(n 34)$. In addition, subjects ( $n$ 113) who under- or over-reported energy intakes $(<3347 \mathrm{~kJ} / \mathrm{d}(<800 \mathrm{kcal} / \mathrm{d})$ or $>17573 \mathrm{~kJ} / \mathrm{d}(>4200 \mathrm{kcal} / \mathrm{d})$, respectively) and those with missing data on covariates ( $n$ 52) were excluded; some individuals fell into more than one exclusion category. To evaluate the incidence, we also excluded subjects who had CKD at baseline ( $n$ 360). Finally, 1630 participants were followed up until survey $\mathrm{V}$ (response rate: $87 \%$ ), with a median duration of $6 \cdot 1$ years $(25-75$ interquartile range: $5 \cdot 6-6 \cdot 5$; Fig. 1).

The ethics committee of the Research Institute for Endocrine Sciences of Shahid Beheshti University of Medical Sciences approved the study protocol and written informed consent was obtained from all participants.

\section{Measurements}

Dietary measurements. Habitual dietary intakes were assessed using a valid and reliable semi-quantitative FFQ by expert interviewers ${ }^{(19-21)}$. Trained dietitians, during face-to-face interviews, asked participants to designate their consumption frequency for each food item consumed during the previous year on a daily, weekly and monthly basis. As the Iranian food composition table (FCT) is incomplete, the United States Department of Agriculture (USDA) FCT was used. For national foods not listed in the USDA FCT, the Iranian FCT was the alternative. We calculated total dietary fibre, as well as fibre contribution from cereals, legumes, vegetables and fruits.

The reliability and validity of the FFQ, which were evaluated against twelve 24-h dietary recalls and two FFQ in a previous study, indicated that the FFQ provides reasonably valid measures of the average long-term dietary fibre intake ${ }^{(19-21)}$.

Measurement of covariates. Information on physical activity was collected by using the modifiable activity questionnaire (MAQ) to calculate metabolic equivalent task (MET)-minutes

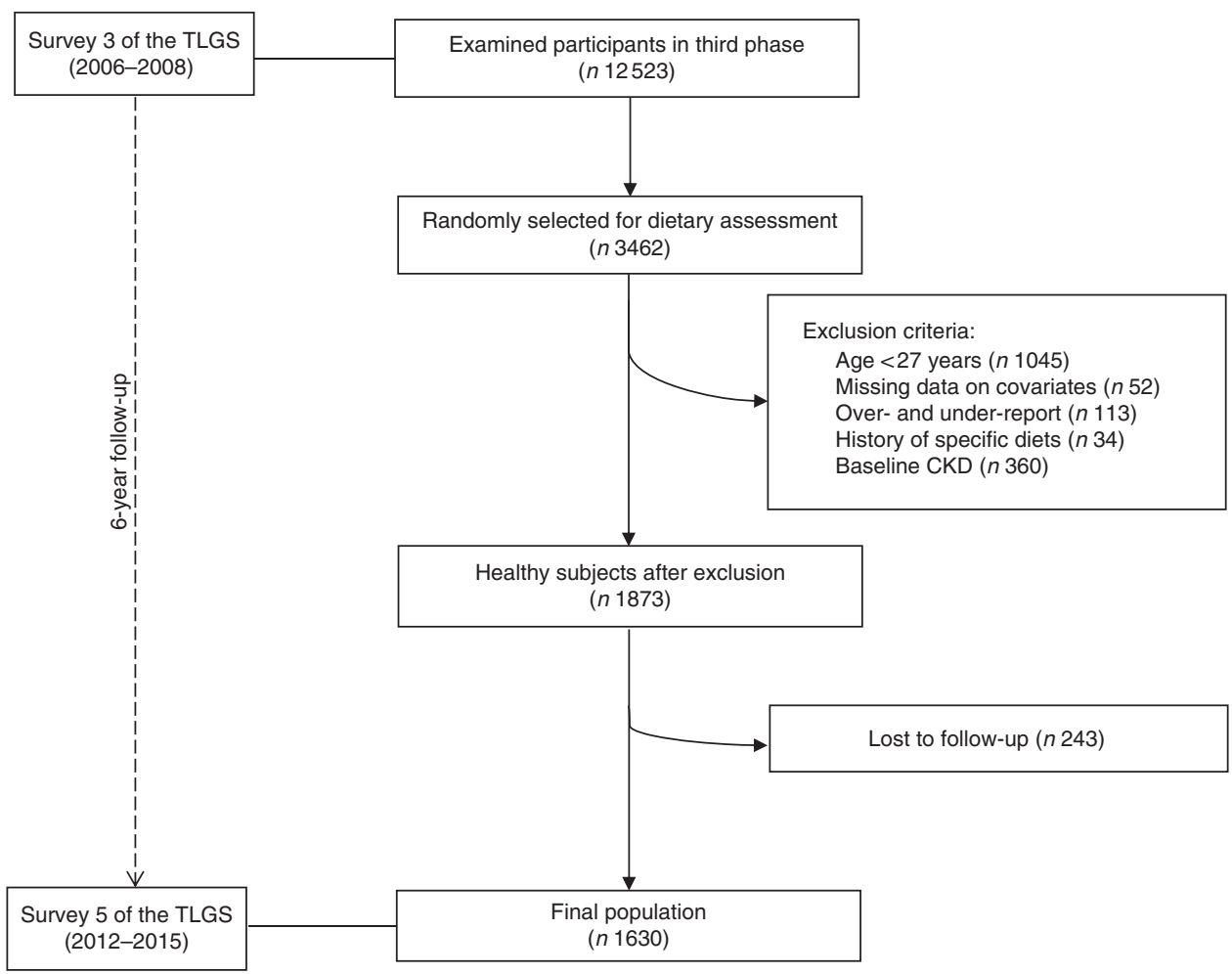

Fig. 1. Flow chart of the Tehran Lipid and Glucose Study (TLGS) participants. CKD, chronic kidney disease. 
per week. High reliability (98\%) and moderate validity (47\%) were found for the Persian translation of $\mathrm{MAQ}^{(22)}$. Low levels of physical activity were considered as MET $<600 \mathrm{~min} /$ week. Weight was recorded in light clothing to the nearest $0.1 \mathrm{~kg}$ on a SECA digital weighing scale (Seca 707; Seca Corporation; range $0 \cdot 1-150 \mathrm{~kg}$ ) and height was measured without shoes to the nearest $0 \cdot 1 \mathrm{~cm}$. BMI was calculated as weight $(\mathrm{kg})$ divided by square of height $\left(\mathrm{m}^{2}\right)$. Arterial blood pressure was measured manually, using a mercury sphygmomanometer with a suitable cuff size for each participant after a 15-min rest in the supine position. Systolic blood pressure (SBP) was determined by the onset of the tapping Korotkoff sound, whereas diastolic blood pressure (DBP) was determined as the disappearance of the Korotkoff sound. Blood pressure was measured twice and the average was considered as the participant's blood pressure.

Blood samples were taken from all participants at the TLGS research laboratory after an overnight fast of $12-14 \mathrm{~h}$. Fasting plasma glucose (FPG) and 2-h plasma glucose (equivalent to $75 \mathrm{~g}$ anhydrous glucose; Cerestar EP) were assayed by enzymatic colorimetric method using glucose oxidase, with both inter- and intra-assay $\mathrm{CV}$ being $<2 \%$. Serum creatinine was measured according to the standard colorimetric Jaffe_Kinetic reaction method at baseline (2006-2008) and after 6 years of follow-up (2012-2015). Both intra- and inter-assay CV were below 3.1\%; all analyses were performed using commercial kits (Pars Azmoon Inc.).

\section{Definitions}

Hypertension was defined as SBP/DBP $\geq 140 / 90 \mathrm{mmHg}$ or current therapy for a definite diagnosis of hypertension ${ }^{(23)}$. Diabetes was defined according to the criteria of the American Diabetes Association as $\mathrm{FPG} \geq 7.0 \mathrm{mmol} / \mathrm{l}$ or 2 -h post $75-\mathrm{g}$ glucose load $\geq 11.1 \mathrm{mmol} / 1$ or current therapy for a definite diagnosis of diabetes ${ }^{(24)}$. We used the Modification of Diet in Renal Disease (MDRD) equation formula to express eGFR in $\mathrm{ml} / \mathrm{min}$ per $1.73 \mathrm{~m}^{2}$ of body surface area ${ }^{(25)}$. The abbreviated MDRD study equation is as follows:

$\mathrm{eGFR}=186 \times(\text { serum creatinine })^{-1.154} \times(\text { age })^{-0.203} \times(0.742$ if female $) \times(1.210$ if African-American $)$

Patients were classified based on their eGFR levels by the National Kidney Foundation guidelines ${ }^{(26)}$; eGFR $\geq 60 \mathrm{ml} / \mathrm{min}$ per $1.73 \mathrm{~m}^{2}$ as not having CKD and eGFR $<60 \mathrm{ml} / \mathrm{min}$ per $1.73 \mathrm{~m}^{2}$ as having CKD.

\section{Statistical analysis}

All data were analysed using the Statistical Package for the Social Sciences program (SPSS) (version 15.0; SPSS Inc.) and $P$ values $<0.05$ were considered statistically significant. Total dietary intake of fibre was categorised into the tertile cut-off points as $\leq 17 \cdot 7,17 \cdot 8-26 \cdot 0$ and $>26 \cdot 1 \mathrm{~g} / \mathrm{d}$; dietary fibre from fruits, vegetables, cereals and legumes was categorised into three groups according to the tertiles of the distribution among the total population. Continuous variables were reported as a mean \pm standard deviation and categorical variables as percentages. Tests of a trend for continuous variables across tertiles of the total fibre intake were conducted using ANOVA and for categorical variables $\chi^{2}$ test was used.
The OR and $95 \%$ CI for the incidence of CKD according to tertiles of dietary exposure was assessed with multivariable logistic regression models. In this analysis, the first tertile of dietary exposure was considered as the reference category. To calculate the trend of OR across increasing tertiles of dietary exposure, the median values of each fibre category were considered as continuous variables. Three models were considered to adjust potential confounders (model 1, crude (without any covariate); model 2, age (continuous), sex, smoking (yes/no), total energy intake (continuous), physical activity (low, moderate, heavy); and model 3, diabetes (yes/no) and using angiotensin-converting-enzyme inhibitor (yes/no)). As a further adjustment for intakes of dietary fat, $n-3$ fatty acids, $\mathrm{K}, \mathrm{Mg}$ and hypertension did not change the relations substantially, these covariates were not considered in the final models.

\section{Results}

We recorded $220(13.5 \%)$ cases of incident CKD, with a range of eGFR between 29 and $59 \mathrm{ml} / \mathrm{min}$ per $1.73 \mathrm{~m}^{2}$, after 6.1 years of follow-up. Mean age and total fibre intake of participants was 42.8 (SD 11.2) years and 23.8 (sD 11.7) g/d. General characteristics of study participants across tertiles of total fibre intake are presented in Table 1. Participants in the highest, compared with the lowest, tertile of total fibre intake were less likely to be women $(P<0.05)$. No significant differences were found by means of age, BMI and eGFR and prevalence of low physical activity, current smoker, diabetes, hypertension and antihypertensive drug across tertiles of total fibre intake.

Dietary intakes of participants across tertile categories of total fibre intake are shown in Table 2. Participants in the top tertile of total fibre intake had higher consumption of protein, plant protein, carbohydrate and $\mathrm{K}$ than those in the bottom tertile $(P<0.05)$; however, consumption of animal protein, total and SFA tended to decrease across tertiles of total fibre intake $(P<0 \cdot 05)$.

In the crude model, total fibre intake was inversely associated with an incidence of CKD (Table 3). After adjustment for age, sex, smoking, total energy intake, physical activity, diabetes and using angiotensin-converting-enzyme inhibitor, the OR for subjects in the highest compared with the lowest tertile of total fibre intake was 0.47 (95\% CI 0.27, 0.86). A significant decreasing linear trend was noted across tertiles of total fibre intake for risk of incident CKD $\left(P_{\text {for trend }}<0 \cdot 001\right)$. In addition, the risk of incident CKD decreased by $11 \%$ for every $5 \mathrm{~g} / \mathrm{d}$ increase in total fibre intake.

We also examined the association between various sources of dietary fibre and incidence of CKD. After adjusting for potential confounders, OR for participants in the highest compared with the lowest tertile of fibre from vegetables was 0.63 (95\%CI $0.43,0.93$ ) and from legumes it was 0.68 (95\% CI 0.47 , 0.98). In addition, a significant decreasing linear trend was noted across tertiles of dietary fibre from vegetables and legumes for the risk of incident CKD $\left(P_{\text {for trend }}<0 \cdot 05\right)$. The risk of incident CKD decreased $50 \%$ for every $5 \mathrm{~g} / \mathrm{d}$ increase in dietary fibre from legumes. Furthermore, there was no significant association of fibre from fruits and cereals with the risk of incident CKD. 
Table 1. Baseline characteristics of participants according to tertiles $(T)$ of total fibre intake (Mean values and standard deviations; percentages)

\begin{tabular}{|c|c|c|c|c|c|c|c|}
\hline & \multicolumn{6}{|c|}{ Tertiles of total fibre } & \multirow[b]{3}{*}{$P_{\text {for trend }}{ }^{\star}$} \\
\hline & \multicolumn{2}{|c|}{$\mathrm{T} 1(n 544)$} & \multicolumn{2}{|c|}{ T2 ( $n$ 543) } & \multicolumn{2}{|c|}{ T3 $(n$ 543) } & \\
\hline & Mean & SD & Mean & SD & Mean & SD & \\
\hline Median total fibre $(\mathrm{g} / \mathrm{d})$ & \multicolumn{2}{|c|}{$13 \cdot 6$} & \multicolumn{2}{|c|}{21.5} & \multicolumn{2}{|c|}{33.5} & \\
\hline Age (years) & $42 \cdot 4$ & $11 \cdot 1$ & $42 \cdot 8$ & 11.2 & $43 \cdot 3$ & 11.4 & 0.190 \\
\hline Women $(\%)$ & \multicolumn{2}{|c|}{$56 \cdot 8$} & \multicolumn{2}{|c|}{$49 \cdot 2$} & \multicolumn{2}{|c|}{$45 \cdot 5$} & 0.001 \\
\hline BMI $\left(\mathrm{kg} / \mathrm{m}^{2}\right)$ & $27 \cdot 2$ & 4.5 & $27 \cdot 6$ & 4.5 & 27.9 & 4.6 & 0.061 \\
\hline Current smoker (\%) & \multicolumn{2}{|c|}{9.9} & \multicolumn{2}{|c|}{$11 \cdot 2$} & \multicolumn{2}{|c|}{$10 \cdot 1$} & 0.750 \\
\hline Low physical activity (\%) & \multirow{2}{*}{\multicolumn{2}{|c|}{$\begin{array}{c}71.5 \\
8.1\end{array}$}} & \multicolumn{2}{|c|}{$69 \cdot 4$} & \multicolumn{2}{|c|}{$69 \cdot 2$} & 0.282 \\
\hline Diabetes (\%) & & & \multicolumn{2}{|c|}{$7 \cdot 6$} & \multicolumn{2}{|c|}{$8 \cdot 8$} & 0.738 \\
\hline Hypertension (\%) & \multicolumn{2}{|c|}{11.6} & \multirow{2}{*}{\multicolumn{2}{|c|}{$\begin{array}{c}13 \cdot 4 \\
3 \cdot 3\end{array}$}} & \multicolumn{2}{|c|}{12.5} & 0.635 \\
\hline Antihypertensive drug use (\%) & \multicolumn{2}{|c|}{$3 \cdot 1$} & & & \multirow{2}{*}{\multicolumn{2}{|c|}{$\begin{array}{l}2.2 \\
1.3\end{array}$}} & 0.564 \\
\hline Angiotensin-converting-enzyme inhibitor use (\%) & \multicolumn{2}{|c|}{1.7} & \multicolumn{2}{|c|}{2.0} & & & 0.636 \\
\hline eGFR $\left(\mathrm{ml} / \mathrm{min}\right.$ per $\left.1.73 \mathrm{~m}^{2}\right)$ & 73.4 & $8 \cdot 7$ & 73.3 & 8.4 & 74.3 & 8.6 & 0.066 \\
\hline
\end{tabular}

eGFR, estimated glomerular filtration rate.

* Tests of trend for continuous variables across tertiles of total fibre intake were conducted using ANOVA, and for categorical variables $\chi^{2}$ test was used.

Table 2. Baseline dietary intakes of participants, according to tertiles (T) of total fibre intake

\begin{tabular}{|c|c|c|c|c|c|c|c|c|c|}
\hline & \multirow{2}{*}{\multicolumn{2}{|c|}{ Total population }} & \multicolumn{6}{|c|}{ Tertiles of the total fibre } & \multirow[b]{3}{*}{$P_{\text {for trend }}{ }^{*}$} \\
\hline & & & \multicolumn{2}{|c|}{$\mathrm{T} 1(n 544)$} & \multicolumn{2}{|c|}{ T2 ( $n$ 543) } & \multicolumn{2}{|c|}{ T3 $(n 543)$} & \\
\hline & Mean & SD & Mean & SD & Mean & SD & Mean & SD & \\
\hline Total energy (kJ) & 9703 & 3423 & 7243 & 1833 & 9259 & 2272 & 12606 & 3351 & $<0.001$ \\
\hline Total energy (kcal) & 2319 & 818 & 1731 & 438 & 2213 & 543 & 3013 & 801 & $<0.001$ \\
\hline Protein (\% energy) & 13.7 & $2 \cdot 4$ & 13.5 & $2 \cdot 6$ & 13.7 & $2 \cdot 2$ & $13 \cdot 9$ & $2 \cdot 4$ & $<0.001$ \\
\hline Animal protein $(g / 1000)$ & 18.5 & $7 \cdot 0$ & 19.7 & 7.6 & $18 \cdot 6$ & $6 \cdot 1$ & $17 \cdot 4$ & $7 \cdot 2$ & $<0.001$ \\
\hline Plant protein $(g / 1000)$ & $15 \cdot 9$ & $4 \cdot 2$ & $14 \cdot 3$ & $3 \cdot 3$ & $15 \cdot 9$ & 3.5 & $17 \cdot 6$ & 4.8 & $<0.001$ \\
\hline Carbohydrate (\% energy) & $57 \cdot 7$ & $7 \cdot 2$ & 55.5 & $7 \cdot 3$ & 57.8 & $6 \cdot 7$ & 59.8 & 6.9 & $<0.001$ \\
\hline Total fat (\% energy) & $31 \cdot 2$ & $7 \cdot 1$ & 32.8 & 7.6 & 31.1 & 6.9 & 29.6 & 6.4 & $<0.001$ \\
\hline SFA (\% energy) & $10 \cdot 5$ & $5 \cdot 5$ & 11.5 & 8.6 & $10 \cdot 3$ & $2 \cdot 7$ & 9.6 & $2 \cdot 7$ & $<0.001$ \\
\hline Dietary fibre $(\mathrm{g} / \mathrm{d})$ & 23.8 & 11.7 & $13 \cdot 1$ & $3 \cdot 1$ & 21.6 & $2 \cdot 4$ & $36 \cdot 6$ & $10 \cdot 7$ & $<0.001$ \\
\hline $\mathrm{K}(\mathrm{mg} / \mathrm{d})$ & 3861 & 1792 & 2601 & 794 & 3633 & 976 & 5347 & 2051 & $<0.001$ \\
\hline Cereal fibre (\%) & 38.2 & $16 \cdot 9$ & 36.5 & 14.4 & 37.4 & $16 \cdot 2$ & $38 \cdot 7$ & $19 \cdot 7$ & 0.093 \\
\hline Fruit fibre (\%) & $26 \cdot 3$ & $16 \cdot 9$ & 28.7 & $16 \cdot 2$ & $27 \cdot 5$ & $14 \cdot 1$ & $22 \cdot 7$ & $12 \cdot 7$ & $<0.001$ \\
\hline Vegetable fibre (\%) & $18 \cdot 3$ & 9.5 & 16.9 & 9.8 & 17.9 & 8.4 & $20 \cdot 0$ & $10 \cdot 4$ & $<0.001$ \\
\hline Legume fibre (\%) & $5 \cdot 8$ & $6 \cdot 10$ & $5 \cdot 6$ & $5 \cdot 2$ & $5 \cdot 8$ & $5 \cdot 7$ & $6 \cdot 1$ & $7 \cdot 2$ & 0.017 \\
\hline
\end{tabular}

* Test of trend across tertiles of total fibre intake was conducted using ANOVA.

\section{Discussion}

This population-based prospective study showed that high total fibre intake was related to lower incidence of CKD after $6 \cdot 1$ years of follow-up. Subjects who consumed more than $26 \cdot 0 \mathrm{~g} / \mathrm{d}$ of fibre had $50 \%$ lower risk of CKD occurrence compared with those who consumed $\leq 17.7 \mathrm{~g} / \mathrm{d}$. We also observed an $11 \%$ lower risk of incident CKD per $5-\mathrm{g} / \mathrm{d}$ increase in total fibre intake. Protective associations of specific sources of fibre with the risk of incident CKD were observed for vegetable and legume fibre. No association was observed between the risk of CKD and the cereal or fruit fibre intake.

Relatively limited studies investigated the association of total fibre intake with CKD ${ }^{(11,12,27,28)}$. Data of 2600 participants aged $\geq 50$ years from Blue Mountains Eye Study showed no significant association of fibre intake with the prevalence of moderate $\mathrm{CKD}^{(11)}$. However, among 1110 participants aged 70-71 years, higher intake of total fibre had desirable association with $\mathrm{eGFR}^{(12)}$. In a cross-sectional study of non-diabetic individuals from the PREDIMED study, among subjects in the highest quartile of fibre intake the risk of incident CKD decreased by $42 \%{ }^{(27)}$. In addition, in 5416 subjects, aged $>40$ years, dietary fibre consumption was found to be associated with a reduced risk of albuminuria ${ }^{(28)}$. Besides studies investigating the association of dietary fibre on CKD, there are studies that show other desirable effects of dietary fibre on kidney-related disorders ${ }^{(13,29)}$. A recent meta-analysis showed that total fibre intake was associated with $26 \%$ lower risk of renal cell carcinoma ${ }^{(29)}$. In addition, women with no history of kidney stones in the highest compared with the lowest categories of dietary fibre intake had a $22 \%$ decreased risk of stone formation ${ }^{(13)}$.

In our study, participants in the highest tertile of dietary fibre intake showed lower consumption of animal protein and saturated fat and higher consumption of plant protein and $\mathrm{K}$ in comparison with those in the lowest tertile. Indeed, higher fibre intake was accompanied by healthier dietary behaviour that may affect kidney function; however, there were no differences between participants in the tertiles of fibre intake according to 
Table 3. Incident chronic kidney disease according to the dietary fibre intake and per 5-g increase in intake among participants of the Tehran Lipid and Glucose Study (Odds ratios and $95 \%$ confidence intervals)

\begin{tabular}{|c|c|c|c|c|c|c|c|c|}
\hline & \multicolumn{5}{|c|}{ Tertiles of dietary fibre } & \multirow[b]{3}{*}{$P_{\text {for trend }}{ }^{*}$} & \multirow{2}{*}{\multicolumn{2}{|c|}{ Per 5-g increase }} \\
\hline & \multirow[b]{2}{*}{ T1 } & \multicolumn{2}{|r|}{$\mathrm{T} 2$} & \multicolumn{2}{|c|}{ T3 } & & & \\
\hline & & OR & $95 \% \mathrm{Cl}$ & OR & $95 \% \mathrm{Cl}$ & & OR & $95 \% \mathrm{Cl}$ \\
\hline Total fibre (median) & 13.6 & & 21.5 & & 3.5 & & & \\
\hline Case/total & $81 / 544$ & & $85 / 543$ & & .543 & & & \\
\hline Model $1 \dagger$ & Ref. & 1.06 & $0.76,1.47$ & 0.63 & $0.44,0.91$ & 0.001 & 0.94 & $0.88,1.00$ \\
\hline Model $2 \ddagger$ & Ref. & 0.98 & $0.68,1.39$ & 0.46 & $0.29,0.76$ & 0.001 & 0.88 & $0.81,0.97$ \\
\hline Model 3§ & Ref. & 0.97 & $0.68,1.38$ & 0.47 & $0.28,0.76$ & 0.001 & 0.89 & $0.81,0.97$ \\
\hline Fruit fibre (median) & 1.96 & & 5.14 & & 0.28 & & & \\
\hline Case/total & $75 / 543$ & & $77 / 544$ & & $/ 543$ & & & \\
\hline Model $1 \dagger$ & Ref. & 1.03 & $0.73,1.45$ & 0.89 & $0.63,1.27$ & 0.512 & 0.93 & $0.82,1.07$ \\
\hline Model $2 \ddagger$ & Ref. & 1.01 & $0.71,1.44$ & 0.82 & $0.56,1.20$ & 0.291 & 0.89 & $0.77,1.04$ \\
\hline Model 3§ & Ref. & 1.02 & $0.72,1.46$ & 0.82 & $0.56,1.21$ & 0.312 & 0.89 & $0.77,1.04$ \\
\hline Vegetable fibre (median) & 1.81 & & 3.51 & & 20 & & & \\
\hline Case/total & $79 / 544$ & & $74 / 543$ & & $/ 543$ & & & \\
\hline Model $1 \dagger$ & Ref. & 0.91 & $0.65,1.28$ & 0.84 & $0.59,1.19$ & 0.341 & 0.97 & $0.77,1.22$ \\
\hline Model $2 \ddagger$ & Ref. & 0.76 & $0.53,1.09$ & 0.63 & $0.43,0.93$ & 0.024 & 0.86 & $0.67,1.11$ \\
\hline Model $3 \S$ & Ref. & 0.75 & $0.53,1.08$ & 0.63 & $0.43,0.93$ & 0.026 & 0.87 & $0.67,1.12$ \\
\hline Cereal fibre (median) & 3.83 & & 7.00 & & 3.18 & & & \\
\hline Case/total & $89 / 544$ & & $63 / 543$ & & $3 / 543$ & & & \\
\hline Model $1 \dagger$ & Ref. & 0.67 & $0.67,0.95$ & 0.73 & $0.52,1.03$ & 0.098 & 0.94 & $0.78,1.13$ \\
\hline Model $2 \ddagger$ & Ref. & 0.66 & $0.46,0.94$ & 0.76 & $0.51,1.13$ & 0.211 & 0.98 & $0.80,1.20$ \\
\hline Model $3 \S$ & Ref. & 0.66 & $0.46,0.95$ & 0.75 & $0.50,1.11$ & 0.180 & 0.97 & $0.79,1.20$ \\
\hline Legume fibre (median) & 0.30 & & 0.79 & & 34 & & & \\
\hline Case/total & $86 / 544$ & & $74 / 543$ & & $/ 543$ & & & \\
\hline Model $1 \dagger$ & Ref. & 0.84 & $0.60,1 \cdot 17$ & 0.66 & $0.46,0.94$ & 0.026 & 0.48 & $0.23,0.98$ \\
\hline Model $2 \ddagger$ & Ref. & 0.82 & $0.58,1.16$ & 0.67 & $0.46,0.97$ & 0.035 & 0.51 & $0.22,0.92$ \\
\hline Model $3 \S$ & Ref. & 0.84 & $0.59,1.19$ & 0.68 & $0.47,0.98$ & 0.038 & 0.50 & $0.23,0.92$ \\
\hline
\end{tabular}

Ref., referent values. The tertile range for each category was $\leq 17.7,17.8-26.0$ and $>26.1 \mathrm{~g} / \mathrm{d}$ for total fibre; $\leq 3.5,3.6-7.2$ and $>7.3 \mathrm{~g} / \mathrm{d}$ for fruit fibre; $\leq 2.6$, $2.7-4.5$ and $>4.6 \mathrm{~g} / \mathrm{d}$ for vegetable fibre; $\leq 5.4,5.5-9.4$ and $>9.5 \mathrm{~g} / \mathrm{d}$ for cereal fibre; and $\leq 0.5,0.6-1.2$ and $>1.3 \mathrm{~g} / \mathrm{d}$ for legume fibre.

${ }^{*} P_{\text {for trend }}$ across tertiles calculated with the exposure modelled as a continuous variable.

$\dagger$ Model 1: crude.

‡ Model 2: adjusted for age, sex, smoking, total energy intake, physical activity.

$\S$ Model 3: additionally adjusted for diabetes and using angiotensin-converting-enzyme inhibitor.

the BMI, current smoker, physical activity and anti-hypertensive drug use. In a previous study, we observed that independent of hypertension and diabetes, higher intakes of plant protein and PUFA had a decreasing effect on risk of CKD, whereas animal protein increased the risk of $\mathrm{CKD}^{(8)}$. Furthermore, the foods rich in fibre are the sources of $\mathrm{K}$ as well. $\mathrm{K}$ can bind to organic anions and metabolised to bicarbonate, so that the net rate of endogenous acid production in comparison with the rate of acid production from animal foods decreased ${ }^{(30)}$. Hence, plant-based diet by providing alkali might decrease the risk of $\mathrm{CKD}^{(31)}$.

According to the present study, fibre from vegetables and legumes, but not from fruits and cereals, was significantly associated with incident CKD. We also found that per $5-\mathrm{g} / \mathrm{d}$ increase in intake of fibre from legumes led to $50 \%$ lower risk of incident CKD. Gopinath et $a l^{(11)}$ observed that participants in the highest compared with those in the lowest quartile of dietary cereal fibre intake had a $50 \%$ reduced risk of incident CKD; however, there were no significant findings between fibre from fruits and vegetables and the risk of CKD. Our results on kidney function are consistent with a previous meta-analysis that showed that the risk of renal cell carcinoma was inversely associated with legume and vegetable fibre intake, but not with fibre intake from fruits and cereals ${ }^{(29)}$. Dietary intake of legumes decreased the risk of incident CKD by $17 \%$, after 23 years of follow-up ${ }^{(32)}$. People with higher scores of the diet rich in vegetables and legumes such as the Mediterranean diet had $51 \%$ less risk of developing $\mathrm{CKD}^{(14)}$. Therefore, on the basis of the findings from our study and others, health benefits of fibre intake on kidney function may depend on the food source of dietary fibre. A previous report on cardiovascular outcomes has similarly shown the effects of fibre from different food sources $^{(33)}$.

In our study, we observed that, contrary to the dietary fibre from vegetables and legumes that simultaneously increased with total dietary fibre, intakes of fibre from cereals and fruits did not have a similar trend with that of total dietary fibre intake. Therefore, we found no significant relation of cereals and fruits with the incidence of CKD.

The beneficial effects of dietary fibre from legumes and vegetables on kidney function can be explained by several potential factors: first, dietary fibre from legumes may decrease the glycaemic index of consumer foods leading to a delay in the postprandial glycaemia ${ }^{(34)}$. Second, dietary fibre sources (vegetables and legumes) are also rich in antioxidants and vitamins. Third, vegetable and legume fibre consumption can decrease the risk of incident CKD by attenuating known risk factors such as diabetes, hypertension, hyperlipidaemia 
and low-grade inflammation ${ }^{(35-37)}$. Higher dietary fibre intake was inversely associated with improved glycaemic control, increased insulin sensitivity and reduced risk of type 2 diabetes, all of which are well known for their harmful effects on kidney function $^{(38,39)}$. Another potential risk factor for CKD is hypertension. The blood pressure-lowering effect of dietary fibre was demonstrated in a recent meta-analysis ${ }^{(40)}$.

There are some limitations to this study that need to be mentioned. First, as in most epidemiological studies, our definition of CKD is based on a limited number of isolated creatinine measurements that were not repeated within 3 months to confirm a chronic reduction in GFR. Second, despite controlling for various confounders in our analysis, residual confounding due to unknown or unmeasured confounders cannot be excluded.

Of the study's noteworthy strengths are the use of a complex validated 168-question FFQ and a prospective cohort design with high-quality data and low loss to follow-up. In addition, unlike previous studies, the present study provided data based on habitual dietary intakes in a population-based sample of participants, thereby increasing the generalisability of its results.

We observed inverse associations between total fibre intake and risk of incident CKD - results that show that high fibre intake, mainly from legumes and vegetables, may reduce the occurrence of CKD. This study provides important insight into the beneficial effects of the nutritional management for prevention of CKD and the desirable effects of dietary fibre intake in a Middle-Eastern population that is distinctly different from developed countries. Prospective studies in other populations are needed to confirm these associations and provide the evidence needed to translate these findings into clinical results.

\section{Acknowledgements}

The authors express their appreciation to the participants of the Tehran Lipid and Glucose Study for their enthusiastic support, and the staff of the Tehran Lipid and Glucose Study Unit of the Research Institute for Endocrine Sciences for their valuable help. The authors would like to acknowledge Ms Niloofar Shiva for critical editing of English grammar and syntax of the manuscript.

This work was funded by a grant from the Research Institute for Endocrine Sciences, Shadid Beheshti University of Medical Sciences, Tehran, Iran.

G. A., S. S and E. Y. designed the research; E. Y. analysed and interpreted data; and G. A., S. S. and E. Y. drafted the initial manuscript. P. M. and F. A. supervised the project and approved the final version of the manuscript to be submitted. All authors read and approved the final manuscript.

The authors declare that there are no conflicts of interest.

\section{References}

1. Xu HSP, Ärnlöv J, Banerjee T, et al. (2015) A proinflammatory diet is associated with systemic inflammation and reduced kidney function in elderly adults. $J$ Nutr 145, 729-735.

2. Jha V, Garcia-Garcia G, Iseki K, et al. (2013) Chronic kidney disease: global dimension and perspectives. Lancet 382, 260-272.
3. Hakemi MS (2014) Chronic kidney disease epidemiology. Iran J Kidney Dis 8, 261-262.

4. Levin A, Tonelli M, Bonventre J, et al. (2017) Global kidney health 2017 and beyond: a roadmap for closing gaps in care, research, and policy. Lancet 39, 1888-1917.

5. Tohidi M, Hasheminia M, Mohebi R, et al. (2012) Incidence of chronic kidney disease and its risk factors, results of over 10 year follow up in an Iranian cohort. PLOS ONE 7, e45304.

6. Kazancioglu R (2013) Risk factors for chronic kidney disease: an update. Kidney Int Suppl 3, 368-371.

7. Farhadnejad H, Asghari G, Mirmiran P, et al. (2016) Micronutrient intakes and incidence of chronic kidney disease in adults: Tehran Lipid and Glucose Study. Nutrients 8, 217.

8. Yuzbashian E, Asghari G, Mirmiran P, et al. (2015) Associations of dietary macronutrients with glomerular filtration rate and kidney dysfunction: Tehran Lipid and Glucose Study. J Nephrol 28, 173-180.

9. King DE (2005) Dietary fiber, inflammation, and cardiovascular disease. Mol Nutr Food Res 49, 594-600.

10. Lattimer JM \& Haub MD (2010) Effects of dietary fiber and its components on metabolic health. Nutrients 2, 1266-1289.

11. Gopinath B, Harris DC, Flood VM, et al. (2011) Carbohydrate nutrition is associated with the 5-year incidence of chronic kidney disease. J Nutr 141, 433-439.

12. $\mathrm{Xu} \mathrm{H}$, Huang $\mathrm{X}$, Riserus U, et al. (2014) Dietary fiber, kidney function, inflammation, and mortality risk. Clin J Am SoC Nephrol 9, 2104-2110.

13. Sorensen MD, Hsi RS, Chi T, et al. (2014) Dietary intake of fiber, fruit and vegetables decreases the risk of incident kidney stones in women: a Women's Health Initiative report. $J$ Urol 192, 1694-1699.

14. Asghari G, Farhadnejad H, Mirmiran P, et al. (2017) Adherence to the Mediterranean diet is associated with reduced risk of incident chronic kidney diseases among Tehranian adults. Hypertens Res 40, 96-102.

15. Asghari G, Yuzbashian E, Mirmiran P, et al. (2017) The association between Dietary Approaches to Stop Hypertension and incidence of chronic kidney disease in adults: the Tehran Lipid and Glucose Study. Nephrol Dial Transplant 32, ii224-ii230.

16. Mirmiran P, Bahadoran Z, Khalili Moghadam S, et al. (2016) A prospective study of different types of dietary fiber and risk of cardiovascular disease: Tehran Lipid and Glucose Study. Nutrients 8, 686-697.

17. Hosseinpour-Niazi S, Mirmiran P, Mirzaei S, et al. (2015) Cereal, fruit and vegetable fibre intake and the risk of the metabolic syndrome: a prospective study in the Tehran Lipid and Glucose Study. J Hum Nutr Diet 28, 236-245.

18. Azizi F, Ghanbarian A, Momenan AA, et al. (2009) Prevention of non-communicable disease in a population in nutrition transition: Tehran Lipid and Glucose Study phase II. Trials 10, 5.

19. Asghari G, Rezazadeh A, Hosseini-Esfahani F, et al. (2012) Reliability, comparative validity and stability of dietary patterns derived from an FFQ in the Tehran Lipid and Glucose Study. Br J Nutr 108, 1109-1117.

20. Esfahani FH, Asghari G, Mirmiran P, et al. (2010) Reproducibility and relative validity of food group intake in a food frequency questionnaire developed for the Tehran Lipid and Glucose Study. J Epidemiol 20, 150-158.

21. Mirmiran P, Esfahani FH, Mehrabi Y, et al. (2010) Reliability and relative validity of an FFQ for nutrients in the Tehran Lipid and Glucose Study. Public Health Nutr 13, 654-662.

22. Momenan AA, Delshad M, Sarbazi N, et al. (2012) Reliability and validity of the Modifiable Activity Questionnaire (MAQ) in an Iranian urban adult population. Arch Iran Med 15, 279-282. 
23. Lenfant C, Chobanian AV, Jones DW, et al. (2003) Seventh report of the Joint National Committee on the Prevention, Detection, Evaluation, and Treatment of High Blood Pressure (JNC 7): resetting the hypertension sails. Hypertension $\mathbf{4 1}$, 1178-1179.

24. (2003) Report of the expert committee on the diagnosis and classification of diabetes mellitus. Diabetes Care 26, Suppl. 1, S5-S20.

25. Levey AS, Bosch JP, Lewis JB, et al. (1999) A more accurate method to estimate glomerular filtration rate from serum creatinine: a new prediction equation. Modification of Diet in Renal Disease Study Group. Ann Intern Med 130, 461-470.

26. (2002) K/DOQI clinical practice guidelines for chronic kidney disease: evaluation, classification, and stratification. $\mathrm{Am} \mathrm{J}$ Kidney Dis 39, S1-266.

27. Diaz-Lopez A, Bullo M, Basora J, et al. (2013) Cross-sectional associations between macronutrient intake and chronic kidney disease in a population at high cardiovascular risk. Clin Nutr 32, 606-612.

28. Metcalf PA, Baker JR, Scragg RK, et al. (1993) Dietary nutrient intakes and slight albuminuria in people at least 40 years old. Clin Chem 39, 2191-2198.

29. Huang TB, Ding PP, Chen JF, et al. (2014) Dietary fiber intake and risk of renal cell carcinoma: evidence from a meta-analysis. Med Oncol 31, 125.

30. Phisitkul S, Khanna A, Simoni J, et al. (2010) Amelioration of metabolic acidosis in patients with low GFR reduced kidney endothelin production and kidney injury, and better preserved GFR. Kidney Int 77, 617-623.

31. Mirmiran P, Yuzbashian E, Bahadoran Z, et al. (2016) Dietary acid-base load and risk of chronic kidney disease in adults: Tehran Lipid and Glucose Study. Iran J Kidney Dis 10, 119-125.
32. Haring B, Selvin E, Liang M, et al. (2017) Dietary protein sources and risk for incident chronic kidney disease: results from the Atherosclerosis Risk in Communities (ARIC) Study. J Ren Nutr 27, 233-242.

33. Kan H, Stevens J, Heiss G, et al. (2007) Dietary fiber intake and retinal vascular caliber in the Atherosclerosis Risk in Communities Study. Am J Clin Nutr 86, 1626-1632.

34. Trinidad TP, Mallillin AC, Loyola AS, et al. (2010) The potential health benefits of legumes as a good source of dietary fibre. Br J Nutr 103, 569-574.

35. Ma Y, Hebert JR, Li W, et al. (2008) Association between dietary fiber and markers of systemic inflammation in the Women's Health Initiative Observational Study. Nutrition 24, 941-949.

36. Satija A \& Hu FB (2012) Cardiovascular benefits of dietary fiber. Curr Atheroscler Rep 14, 505-514.

37. Levey AS, Coresh J, Balk E, et al. (2003) National Kidney Foundation practice guidelines for chronic kidney disease: evaluation, classification, and stratification. Ann Intern Med 139, 137-147.

38. Yao B, Fang H, Xu W, et al. (2014) Dietary fiber intake and risk of type 2 diabetes: a dose-response analysis of prospective studies. Eur J Epidemiol 29, 79-88.

39. Fujii H, Iwase M, Ohkuma $\mathrm{T}$, et al. (2013) Impact of dietary fiber intake on glycemic control, cardiovascular risk factors and chronic kidney disease in Japanese patients with type 2 diabetes mellitus: the Fukuoka Diabetes Registry. Nutr J 12, 159.

40. Whelton SP, Hyre AD, Pedersen B, et al. (2005) Effect of dietary fiber intake on blood pressure: a meta-analysis of randomized, controlled clinical trials. J Hypertens 23, 475-481. 